\title{
Identification of potential therapeutic targets for colorectal cancer by bioinformatics analysis
}

\author{
MING YAN, MAOMIN SONG, RIXING BAI, SHI CHENG and WENMAO YAN \\ Department of General Surgery, Beijing Tiantan Hospital, Capital Medical University, Beijing 100050, P.R. China
}

Received May 27, 2015; Accepted October 4, 2016

DOI: $10.3892 / 01.2016 .5328$

\begin{abstract}
The aim of the present study was to identify potential therapeutic targets for colorectal cancer (CRC). The gene expression profile GSE32323, containing 34 samples, including 17 specimens of CRC tissues and 17 of paired normal tissues from CRC patients, was downloaded from the Gene Expression Omnibus database. Following data preprocessing using the Affy and preprocessCore packages, the differentially-expressed genes (DEGs) between the two types of samples were identified with the Linear Models for Microarray Analysis package. Next, functional and pathway enrichment analysis of the DEGs was performed using the Database for Annotation Visualization and Integrated Discovery. The protein-protein interaction (PPI) network was established using the Search Tool for the Retrieval of Interacting Genes database. Utilizing WebGestalt, the potential microRNAs (miRNAs/miRs) of the DEGs were screened and the integrated miRNA-target network was built. A cohort of 1,347 DEGs was identified, the majority of which were mainly enriched in cell cycle-related biological processes and pathways. Cyclin-dependent kinase 1 (CDK1), cyclin B1 (CCNB1), MAD2 mitotic arrest deficient-like $1(M A D 2 L 1)$ and BUB1 mitotic checkpoint serine/threonine kinase $\mathrm{B}(B U B 1 B)$ were prominent in the PPI network, while the over-represented genes in the integrated miRNA-target network were SRY (sex determining region Y)-box 4 (SOX4; targeted by hsa-mir-129), v-myc avian myelocytomatosis viral oncogene homolog (MYC; targeted by hsa-let-7c and hsa-mir-145) and cyclin D1 (CCND1; targeted by hsa-let-7b). CDK1, CCNB1 and CCND1 were also associated with the p53 signaling pathway. Overall, several genes associated with the cell cycle and p53 pathway were identified as biomarkers for CRC. CDK1, CCNB1, $M A D 2 L 1, B U B 1 B, S O X 4$, collagen type I $\alpha 2$ chain and $M Y C$
\end{abstract}

Correspondence to: Dr Maomin Song, Department of General Surgery, Beijing Tiantan Hospital, Capital Medical University, 6 Tiantan Xili, Dongcheng, Beijing 100050, P.R. China

E-mail:maominsss@163.com

Key words: colorectal cancer, differentially-expressed gene, protein-protein interaction network, miRNA-target network, cell cycle, p53 pathway may play significant roles in CRC progression by affecting the cell cycle-related pathways, while $C D K 1, C C N B 1$ and $C C N D 1$ may serve as crucial regulators in the p53 signaling pathway. Furthermore, SOX4, MYC and CCND1 may be targets of miR-129, hsa-mir-145 and hsa-let-7c, respectively. However, further validation of these data is required.

\section{Introduction}

Colorectal cancer (CRC) is the third most common cancer type worldwide, with high morbidity and mortality rates (1). Annually, the global incidence of CRC is estimated to be $\sim 1$ million, with 500,000 mortalities (2). Obesity, smoking, diet and a lack of exercise are risk factors associated with CRC (3). Despite advanced detection approaches, including colonoscopy and fecal immunochemical testing in early stage and precancerous lesions (4), the incidence of CRC remains high. In a previous study, in the United States in 2014, a cohort of 136,830 individuals was estimated to be diagnosed with CRC and 50,310 patient (36.8\%) succumbed (5). In China, rapidly increasing incidence and mortality rates of CRC have been detected in past decades (6). Therefore, extensive studies have been conducted to investigate more effective biological therapies for CRC management. The accumulation of mutations in a large number of oncogenes and tumor suppressor genes, which could active or inhibit the pathways critical for the initiation and progression of CRC, were detected (7). Several biomarkers have been established for the detection of metastatic CRC, including KRAS and RAS mutations $(8,9)$. Additionally, the crucial pathways were also observed. Smith et al showed that tumor protein p53 promoted the progression of CRC through the alteration of genetic pathways (10). The nuclear factor- $\kappa \mathrm{B}$ signaling pathway was reported to contribute to the carcinogenesis of CRC (11). MicroRNAs (miRNAs/miRs) are small RNAs that play central roles in cancer development via the regulation of its target genes. The altered expression of miR-21, miR-31, miR-143 and miR-145 was implicated in CRC progression (12). A recent study recruiting a genome-wide screening method identified 16 vital genes in CRC, such as $S C A R A 5$, which was affected by methylation (13). However, the comprehensive regulatory mechanisms of $\mathrm{CRC}$, particularly the interplayed associations between miRNAs and genes, remain obscure. The present study utilized the expression profile data in the study by Khamas et al (13) to identify the differentially-expressed genes (DEGs) between CRC tissues 
and paired normal control tissues. In addition, the interactions amongst the DEGs were further investigated through protein-protein interaction (PPI) network analysis. Furthermore, the miRNAs that targeted the DEGs were also predicted. As a whole, all these bioinformatical analyses were aimed to identify potential biomarkers for the prognosis and prevention of $\mathrm{CRC}$, and to uncover the underlying regulatory mechanism of CRC progression.

\section{Materials and methods}

Gene expression profile data. The gene expression profile data GSE32323, which was deposited by Khamas et al (13), was used. The public Gene Expression Omnibus database (http://www.ncbi.nlm.nih.gov/geo/), was utilized in the study. The platform used was GPL570 (Affymetrix Human Genome U133 Plus 2.0 Array; Agilent Technologies, Palo Alto, CA, USA). In the expression profile, there were 34 samples derived from the $\mathrm{CRC}$ patients, consisting of 17 from cancerous tissues (CRC samples) and 17 from paired normal tissues (control samples).

Identification of DEGs. Following the data preprocessing, including background correction and the transformation from probeleveltogenesymbolusingtheAffypackage(14)inRlanguage (http://www.bioconductor.org/packages/release/bioc/html/affy. html), the data was subjected to normalization with the preprocessCore package (version 1.28.0; http://www.bioconductor. org/packages/3.0/bioc/html/preprocessCore.html) (15). Subsequently, the DEGs between CRC and normal samples were selected basing on a t-test of Linear Models for Microarray Analysis package in $\mathrm{R}$ (version 3.22.7; http://www.bioconductor.org/packages/release/bioc/html/limma.html) (16). The fold-change (FC) of the gene expression was also calculated The threshold criteria for the DEG selection were $\mathrm{P}<0.05$ and $\log 2 \mathrm{FCl} \geq 1$.

Functional enrichment analysis of the DEGs. To investigate the functions and processes that may be altered by the identified DEGs, the Gene ontology (GO) and Kyoto Encyclopedia of Genes and Genomes pathway enrichment analyses were performed, using the online tool of the Database for Annotation Visualization and Integrated Discovery (version 6.7; http://david.abcc.Ncifcrf.gov/) (17), a potent program integrating the gene or protein functional annotations with graphical summary. The cut-off value for the screening of significant functions and pathways was $\mathrm{P}<0.05$.

Establishment of the PPI network. The Search Tool for the Retrieval of Interacting Genes (STRING) database (version 9.1; http://string-db.org/) (18) was recruited to predict the potential interactions amongst the identified DEGs from the protein level. Only the interactions containing at least one DEG were filtered out to build the PPI network, with the criterion of a combined score of $>0.4$, as visualized by Cytoscape (version 3.2.1; http://cytoscape.org/) software (19).

Prediction of targets of microRNAs. Using the web-based gene set analysis toolkit (WebGestalt; Vanderbilt University,
TN, USA; http://bioinfo.vanderbilt.edu/webgestalt/) (20), the regulatory miRNAs of the DEGs were selected.

\section{Results}

DEGs between CRC and normal samples. According to the aforementioned selection criteria, a set of 1,347 DEGs, including 659 upregulated genes and 688 downregulated genes, were identified.

Altered functions and pathways by the DEGs. As indicated in the results of the enrichment analysis (Table I), the upregulated DEGs were significantly enriched in biological processes (BPs) that included the mitotic cell cycle (GO:0000278), nuclear division (GO:0000280) and the cell cycle (GO:0007049), and pathways such as the cell cycle (Hsa04110) and DNA replication (Hsa03030). For the downregulated DEGs, the over-represented functional GO terms were cellular response to zinc ion (GO:0071294), cellular response to chemical stimulus (GO:0070887) and cellular response to chemical stimulus (GO:0070887), while the prominent pathways were metabolic pathways (Hsa01100) and pancreatic secretion (Hsa04972) (Table II).

PPI network of the DEGs. By mapping the DEGs into the STRING database, the potential interactions of the DEGs from the protein level were predicted. As a result, a PPI network comprising 1,478 edges and 462 nodes were established. A protein in the network serves as a 'node', and the 'degree' of a node represents the number of the interactions between two nodes. Based on this definition, the top ten nodes with high degrees in the PPI network were cyclin-dependent kinase 1 (CDK1; degree=59), cyclin B1 (CCNB1; degree=48), NDC80 kinetochore complex component (degree=45), non-SMC condensin I complex, subunit G (degree $=45)$, MAD2 mitotic arrest deficient-like 1 (MAD2L1; degree=44), centromere protein $\mathrm{F}$ (degree=41), BUB1 mitotic checkpoint serine/threonine kinase B (BUB1B; degree=39), centromere protein A (degree=37), PDZ-binding kinase (degree=36) and TPX2, microtubule nucleation factor (degree=36) (Fig. 1).

Integrated miRNA-target regulatory network. Using the WebGestalt software, the integrated miRNA-target network was built, consisting of 459 nodes (305 miRNAs and 154 DEGs) and 646 edges (Fig. 2). In this network, the notable genes that were targeted by multiple miRNAs included SRY (sex determining region Y)-box 4 (SOX4; targeted by 27 miRs, including hsa-mir-129, hsa-mir-133a/b and hsa-mir-204), CCND1 (cyclin D1; targeted by 21 miRs, including hsa-let-7b, hsa-mir-155, hsa-mir-16 and hsa-mir-195) and v-myc avian myelocytomatosis viral oncogene homolog (MYC; targeted by 10 miRs, including hsa-mir-34a, hsa-let-7c, hsa-mir-145 and hsa-mir-24.

\section{Discussion}

CRC is one of the most lethal cancers in the world (3). Biomarker therapeutic methods may be the most effective approaches for the management of CRC. In the present study, a total of 1,347 DEGs (659 upregulated and 
Table I. GO and pathway enrichment analysis of the upregulated DEGs (top 5 in each category, as ranked by the P-value).

\begin{tabular}{|c|c|c|c|c|}
\hline Category & ID & Term & Count & P-value \\
\hline BP & GO:0000278 & Mitotic cell cycle & 102 & $2.63 \times 10^{-24}$ \\
\hline BP & GO:0000280 & Nuclear division & 55 & $2.26 \times 10^{-22}$ \\
\hline BP & GO:0007049 & Cell cycle & 128 & $3.47 \times 10^{-21}$ \\
\hline $\mathrm{BP}$ & GO:0007067 & Mitosis & 55 & $1.25 \times 10^{-18}$ \\
\hline $\mathrm{BP}$ & GO:0022402 & Cell cycle process & 117 & $1.15 \times 10^{-18}$ \\
\hline $\mathrm{CC}$ & GO:0031981 & Nuclear lumen & 140 & $1.68 \times 10^{-17}$ \\
\hline $\mathrm{CC}$ & GO:0044428 & Nuclear region & 158 & $2.32 \times 10^{-16}$ \\
\hline $\mathrm{CC}$ & GO:0043233 & Organelle lumen & 164 & $1.44 \times 10^{-15}$ \\
\hline $\mathrm{CC}$ & GO:0031974 & Membrane-enclosed lumen & 166 & $1.55 \times 10^{-15}$ \\
\hline $\mathrm{CC}$ & GO:0070013 & Intracellular organelle lumen & 161 & $2.22 \times 10^{-15}$ \\
\hline $\mathrm{MF}$ & GO:0005515 & Protein binding & 319 & $2.45 \times 10^{-8}$ \\
\hline MF & GO:0005488 & Binding & 450 & $1.92 \times 10^{-6}$ \\
\hline MF & GO:0003678 & DNA helicase activity & 9 & $2.10 \times 10^{-5}$ \\
\hline MF & GO:0004386 & Helicase activity & 15 & $1.42 \times 10^{-4}$ \\
\hline $\mathrm{MF}$ & GO:0008009 & Chemokine activity & 8 & $1.70 \times 10^{-4}$ \\
\hline KEGG pathway & Hsa04110 & Cell cycle & 24 & $1.21 \times 10^{-11}$ \\
\hline KEGG pathway & Hsa03030 & DNA replication & 11 & $3.64 \times 10^{-8}$ \\
\hline KEGG pathway & Hsa03013 & RNA transport & 21 & $1.19 \times 10^{-7}$ \\
\hline KEGG pathway & Hsa03008 & Ribosome biogenesis in eukaryotes & 15 & $1.60 \times 10^{-7}$ \\
\hline KEGG pathway & Hsa04115 & p53 signaling pathway & 10 & $1.72 \times 10^{-4}$ \\
\hline
\end{tabular}

GO, gene ontology; DEGs, differentially-expressed genes; KEGG, Kyoto Encyclopedia of Genes and Genomes; BP, biological process; CC, cell component; MF, molecular function; Count, numbers of DEGs enriched in each term.

Table II. GO and pathway enrichment analysis of the downregulated DEGs (top 5 in each category, as ranked by the P-value).

\begin{tabular}{|c|c|c|c|c|}
\hline Category & ID & Term & Count & P-value \\
\hline $\mathrm{BP}$ & GO:0071294 & Cellular response to zinc ion & 7 & $2.45 \times 10^{-8}$ \\
\hline $\mathrm{BP}$ & GO:0070887 & Cellular response to chemical stimulus & 112 & $2.90 \times 10^{-7}$ \\
\hline $\mathrm{BP}$ & GO:0010035 & Response to inorganic substance & 32 & $3.18 \times 10^{-7}$ \\
\hline $\mathrm{BP}$ & GO:0006629 & Lipid metabolic process & 77 & $4.91 \times 10^{-7}$ \\
\hline $\mathrm{BP}$ & GO:0050896 & Response to stimulus & 303 & $1.34 \times 10^{-6}$ \\
\hline $\mathrm{CC}$ & GO:0005615 & Extracellular space & 69 & $6.65 \times 10^{-11}$ \\
\hline $\mathrm{CC}$ & GO:0005576 & Extracellular region & 131 & $3.24 \times 10^{-10}$ \\
\hline $\mathrm{CC}$ & GO:0044421 & Extracellular region part & 81 & $1.11 \times 10^{-9}$ \\
\hline $\mathrm{CC}$ & GO:0071944 & Cell periphery & 224 & $1.50 \times 10^{-9}$ \\
\hline $\mathrm{CC}$ & GO:0016020 & Membrane & 346 & $6.02 \times 10^{-9}$ \\
\hline MF & GO:0019955 & Cytokine binding & 12 & $1.47 \times 10^{-6}$ \\
\hline MF & GO:0097367 & Carbohydrate derivative binding & 20 & $9.75 \times 10^{-6}$ \\
\hline MF & GO:0008201 & Heparin binding & 16 & $1.03 \times 10^{-5}$ \\
\hline MF & GO:0005539 & Glycosaminoglycan binding & 18 & $2.74 \times 10^{-5}$ \\
\hline MF & GO:0016616 & $\begin{array}{l}\text { Oxidoreductase activity, acting on the } \mathrm{CH}-\mathrm{OH} \\
\text { group of donors, NAD or NADP as acceptor }\end{array}$ & 14 & $3.79 \times 10^{-5}$ \\
\hline KEGG pathway & Hsa01100 & Metabolic pathways & 69 & $1.21 \times 10^{-4}$ \\
\hline KEGG pathway & Hsa04972 & Pancreatic secretion & 12 & $6.96 \times 10^{-4}$ \\
\hline KEGG pathway & Hsa04960 & Aldosterone-regulated sodium reabsorption & 7 & $1.29 \times 10^{-3}$ \\
\hline KEGG pathway & Hsa00910 & Nitrogen metabolism & 5 & $1.90 \times 10^{-3}$ \\
\hline KEGG pathway & Hsa00232 & Caffeine metabolism & 3 & $2.02 \times 10^{-3}$ \\
\hline
\end{tabular}

GO, gene ontology; DEGS, differentially-expressed genes; KEGG, kyoto encyclopedia of genes and genomes; BP, biological process; CC, cell component; MF, molecular function; Count, numbers of DEGs enriched in each term. 


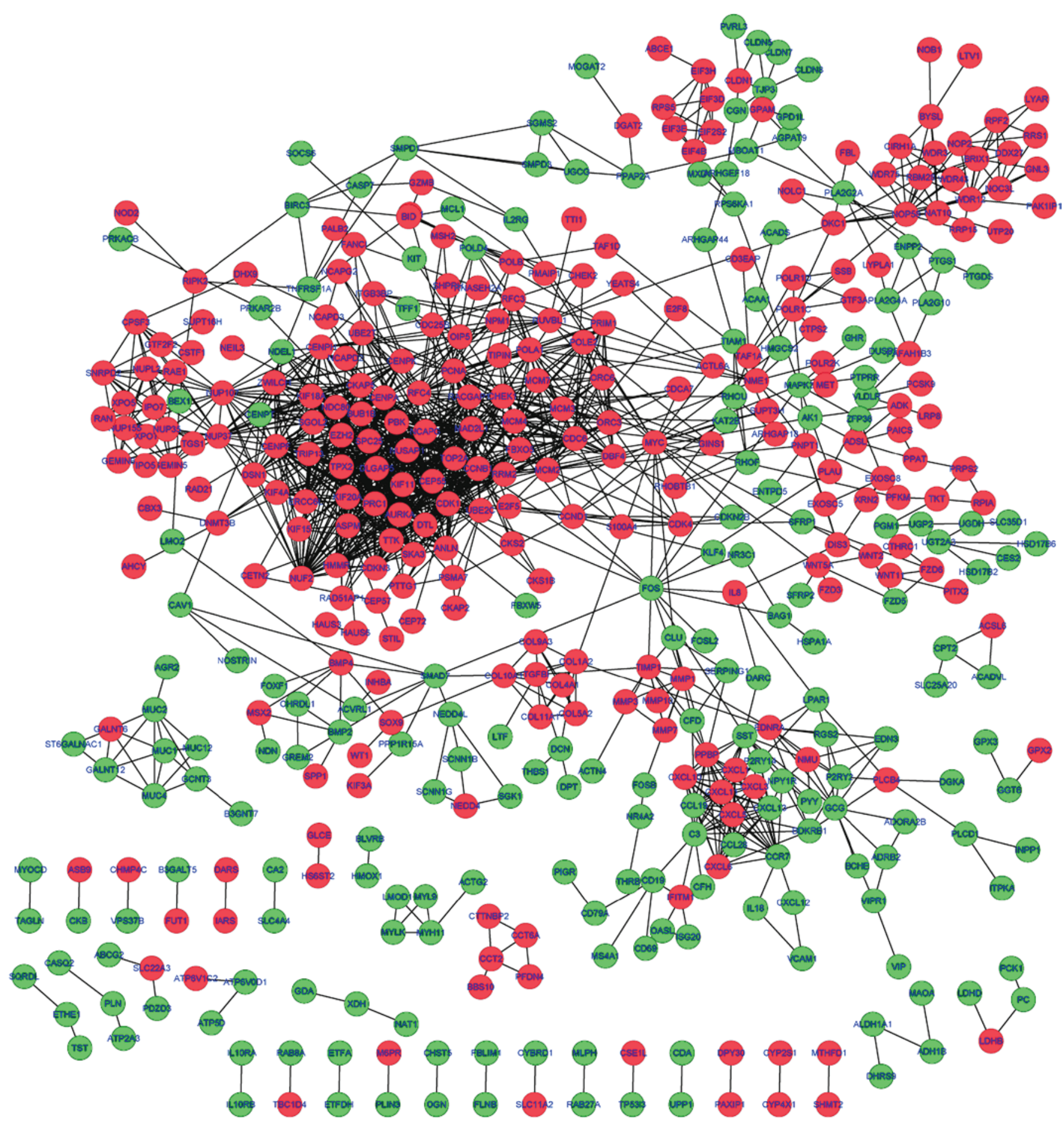

Figure 1. Protein-protein interaction network of the DEGs. Red nodes represent protein products of upregulated DEGs, green nodes represent protein products of downregulated DEGs and the lines between two nodes denote the interactions between them. DEGs, differentially-expressed genes.

688 downregulated) were identified between CRC and normal tissues. Among them, $C D K 1, C C N B 1, M A D 2 L 1$ and $B U B 1 B$, which are mainly enriched in cell cycle-related BPs and pathways, were also the predominant nodes in the PPI network. The integrated miRNA-target network identified crucial genes, including SOX4 (targeted by hsa-mir-129, hsa-mir-133a/b and hsa-mir-204), MYC (targeted by hsa-mir-34a, hsa-let-7c, hsa-mir-145 and hsa-mir-24) and CCND1 (targeted by hsa-let-7b, hsa-mir-155, hsa-mir-16 and hsa-mir-195), which were all enriched in cell cycle-related pathways. $C D K 1, C C N B 1$ and $C C N D 1$ were also associated with the p53 signaling pathways.

Cell cycle-related genes that promote the proliferation of endothelial cells contribute to the progression of tumor growth and metastasis of CRC (21). CDK1 encodes for a serine/threonine kinase that controls the eukaryotic cell cycle by regulating mitotic onset, as well as the centrosome cycle (22). CDK1 promotes cell proliferation via phosphorylation and inhibition of forkhead box $\mathrm{O} 1$ transcription factor (23). The alteration of $C D K 1$ has been found in numerous cancer types, including 




Figure 2. Integrated miRNA-target regulatory network. Red circle nodes represent protein products of upregulated DEGs, green circle nodes represent protein products of downregulated DEGs, pink triangular nodes represent miRNAs and the lines between two nodes denote the interactions between them. DEGs, differentially-expressed genes; miRNA/miR, microRNA.

breast cancer (24), esophageal adenocarcinoma (25) and oral squamous cell carcinoma (26). Deregulated $C D K 1$ has been found in CRC (27), and it has been demonstrated that cantharidin, the traditional Chinese medicine that could induce cell cycle arrest and apoptosis in various cancers, exerted the anticancer function via the inhibition of $C D K 1$ activity (28).
CCNB1 is a regulatory protein involved in mitosis (29). The increased expression of $C C N B 1$ has also been observed in multiple cancer types, including non-small cell lung cancer (29) and gastrointestinal stromal tumors (30). Moreover, $C C N B 1$ serves as a biomarker for the prognosis of estrogen receptor-positive breast cancer (31). CCNB1 plays important 
roles in the cell proliferation at the $\mathrm{G} 2$ phase. It was previously verified that the suppression of $C C N B 1$ by miR-93 resulted in the inhibition of tumor growth in CRC (32).

MAD2L1 and BUB1B are two major mitotic spindle checkpoints. Previous studies considered that the mutation or deficiency in checkpoint proteins may contribute to enhancing the tumor development in breast cancer (33), and the mutation of BUB1, the paralog of BUB1B, was first reported in CRC (34). However, in contrast with these findings, Yuan et al validated the overexpression of $M A D 2 L 1$ and $B U B 1 B$ by reverse transcription-quantitative polymerase chain reaction in breast cancer and proposed that it may alternatively be the overexpression of checkpoint genes that account for genomic instability (35).

The high expression level of SOX4, the transcription factor responsible for the regulation of embryonic development and cell control, was significantly associated with the recurrence of CRC (36). Notably, it was reported that the oncogene SOX4 was regulated by miR-129-2 in endometrial cancer, and that the overexpression of $S O X 4$ was partly caused by the suppression of miR-129-2 (37).

MYC is a central gene that plays important regulatory roles in cell cycle progression. The deficiency of c-MYC inhibited the proliferation of tumor cells in numerous cancer types during the cell cycle through G1 into S phase (38), while the upregulation of MYC transcription by the SNP rs6983267 was demonstrated to promote the development of CRC (39). Moreover, a spectrum of studies has reported the suppression of MYC by miRNAs, including let-7a (40), miR-23a/b (41) and miR-145 (42), in various cancer types. Furthermore, the overexpression of stromal genes, such as collagen type I $\alpha 2$ chain (COL1A2), was also detected in CRC (43).

In the present study, the aforementioned 7 genes were upregulated in CRC samples, and the genes were all enriched in cell cycle-related BP terms and pathways, implying that these genes mediated cell cycle pathways that may play a crucial role in the tumorigenesis and progression of CRC. Combining the previous confirmations with the present predicted miRNA-target interactions, it can be speculated that SOX4 may be the target of miR-129, while MYC may be targeted by hsa-mir-145 and hsa-let-7c.

The $\mathrm{p} 53$ protein acts as a tumor suppressor, as it could prevent DNA damage by promoting cell cycle arrest in the G1 phase or by apoptosis. The alteration of genes in the p53 signaling pathway is tightly correlated with cancer development (44) CCND1 is a cyclin protein that functions as a regulator of CDKs, such as CDK4 or CDK6, during the cell cycle G1/S transition. Amplification of CCNDl has been observed in CRC (45) and the association between increased $C C N D 1$ and the activation of the p53 pathway has been established (46). Besides, the involvement of $C D K 1$ and $C C N B 1$ in the p53 signaling pathway have also been implied $(47,48)$. The present findings indicated that $C D K 1$, CCNB1 and CCND1 were all enriched in the p53 signaling pathway, providing a hint that the three genes may have vital roles in the progression of CRC by the regulation of the p53 signaling pathway. An extensive number of miRNAs downregulated the expression of $C C N D 1$, including miR-193b (49), miR-200b (50), miR-138b (51) and let-7b (52). Based on the correlations in the integrated miRNA-target network, CCNDI was regulated by 21 miRNAs, including hsa-let-7c, suggesting that CCND1 may be the target of hsa-let-7c.
In conclusion, the cell cycle-related pathways mediated by the $C D K 1, C C N B 1, M A D 2 L 1, B U B 1 B, S O X 4, C O L 1 A 2$ and $M Y C$ genes, and the p53 signaling pathway regulated by the $C D K 1, C C N B 1$ and $C C N D 1$ genes may play important roles in the progression of CRC. All these genes may be used as biomarkers for the prognosis of CRC. Furthermore, SOX4 may be targeted by miR-129 and MYC by hsa-mir-145 and hsa-let-7c, while $C C N D 1$ may be the target of hsa-let-7c. However, further experimental validation is warranted to confirm these putative regulatory correlations.

\section{References}

1. Qiu Y, Patwa TH, Xu L, Shedden K, Misek DE, Tuck M, Jin G, Ruffin MT, Turgeon DK, Synal S, et al: Plasma glycoprotein profiling for colorectal cancer biomarker identification by lectin glycoarray and lectin blot. J Proteome Res 7: 1693-1703, 2008.

2. Cajuso T, Hänninen UA, Kondelin J, Gylfe AE, Tanskanen T, Katainen R, Pitkänen E, Ristolainen H, Kaasinen E and Taipale M: Exome sequencing reveals frequent inactivating mutations in ARID1A, ARID1B, ARID2 and ARID4A in microsatellite unstable colorectal cancer. Int J Cancer 135: 611-623, 2014.

3. Mendenhall WM, Amos EH, Rout WR, Zlotecki RA, Hochwald SN and Cance WG: Adjuvant postoperative radiotherapy for colon carcinoma. Cancer 101: 1338-1344, 2004.

4. Quintero E, Castells A, Bujanda L, Cubiella J, Salas D, Lanas Á, Andreu M, Carballo F, Morillas JD, Hernández C, et al: Colonoscopy versus fecal immunochemical testing in colorectal-cancer screening. N Engl J Med 366: 697-706, 2012.

5. Siegel R, Desantis C and Jemal A: Colorectal cancer statistics, 2014. CA Cancer J Clin 64: 104-117, 2014.

6. Huang Z, Huang D, Ni S, Peng Z, Sheng W and Du X: Plasma microRNAs are promising novel biomarkers for early detection of colorectal cancer. Int J Cancer 127: 118-126, 2010.

7. Pino MS and Chung DC: The chromosomal instability pathway in colon cancer. Gastroenterology 138: 2059-2072, 2010.

8. Peeters M, Douillard JY, Van Cutsem E, Siena S, Zhang K, Williams R and Wiezorek J: Mutant KRAS codon 12 and 13 alleles in patients with metastatic colorectal cancer: Assessment as prognostic and predictive biomarkers of response to panitumumab. J Clin Oncol 31: 759-765, 2013.

9. Douillard JY, Oliner KS, Siena S, Tabernero J, Burkes R, Barugel M, Humblet Y, Bodoky G, Cunningham D, Jassem J, et al: Panitumumab-FOLFOX4 treatment and RAS mutations in colorectal cancer. N Engl J Med 369: 1023-1034, 2013.

10. Smith G, Carey FA, Beattie J, Wilkie MJ, Lightfoot TJ, Coxhead J, Garner RC, Steele RJ and Wolf CR: Mutations in APC, Kirsten-ras, and p53-alternative genetic pathways to colorectal cancer. Proc Natl Acad Sci USA 99: 9433-9438, 2002.

11. Wang S, Liu Z, Wang L and Zhang X: NF-kappaB signaling pathway, inflammation and colorectal cancer. Cell Mol Immunol 6: 327-334, 2009.

12. Slaby O, Svoboda M, Fabian P, Smerdova T, Knoflickova D, Bednarikova M, Nenutil R and Vyzula R: Altered expression of miR-21, miR-31, miR-143 and miR-145 is related to clinicopathologic features of colorectal cancer. Oncology 72: 397-402, 2007.

13. Khamas A, Ishikawa T, Shimokawa K, Mogushi K, Iida S, Ishiguro M, Mizushima H, Tanaka H, Uetake H and Sugihara K: Screening for epigenetically masked genes in colorectal cancer Using 5-Aza-2'-deoxycytidine, microarray and gene expression profile. Cancer Genomics Proteomics 9: 67-75, 2012.

14. Gautier L, Cope L, Bolstad BM and Irizarry RA: affy-analysis of Affymetrix GeneChip data at the probe level. Bioinformatics 20 : 307-315, 2004

15. Bolstad BM: Package 'preprocessCore': A collection of pre-processing functions. R Package version 1.28.0, http://www. bioconductor.org/packages/3.0/bioc/html/preprocessCore.html, 2013. Accessed May 7, 2015.

16. Smyth GK: Limma: Linear models for microarray data. In: Bioinformatics and Computational Biology Solutions Using $\{\mathrm{R}\}$ and Bioconductor. Gentleman R, Carey V, Dudoit S, Irizarry R and Huber W (eds.). Springer, New York, pp397-420, 2005. 
17. Dennis G Jr, Sherman BT, Hosack DA, Yang J, Gao W, Lane HC and Lempicki RA: DAVID: Database for annotation, visualization, and integrated discovery. Genome Biol 4: P3, 2003.

18. Franceschini A, Szklarczyk D, Frankild S, Kuhn M, Simonovic M, Roth A, Lin J, Minguez P, Bork P, von Mering C and Jensen LJ: STRING v9.1: Protein-protein interaction networks, with increased coverage and integration. Nucleic Acids Res 41 (Database Issue): D808-D815, 2013.

19. Shannon P, Markiel A, Ozier O, Baliga NS, Wang JT, Ramage D, Amin N, Schwikowski B and Ideker T: Cytoscape: A software environment for integrated models of biomolecular interaction networks. Genome Res 13: 2498-2504, 2003

20. Wang J, Duncan D, Shi Z and Zhang B: WEB-based GEne SeT AnaLysis Toolkit (WebGestalt): Update 2013. Nucleic Acids Res 41: W77-W83, 2013.

21. Hong BS, Cho JH, Kim H, Choi EJ, Rho S, Kim J, Kim JH, Choi DS, Kim YK, Hwang D, et al: Colorectal cancer cell-derived microvesicles are enriched in cell cycle-related mRNAs that promote proliferation of endothelial cells. BMC Genomics 10 $556,2009$.

22. Santamaría D, Barrière C, Cerqueira A, Hunt S, Tardy C, Newton K, Cáceres JF, Dubus P, Malumbres M and Barbacid M: Cdk1 is sufficient to drive the mammalian cell cycle. Nature 448: 811-815, 2007

23. Liu P, Kao TP and Huang H: CDK1 promotes cell proliferation and survival via phosphorylation and inhibition of FOXO1 transcription factor. Oncogene 27: 4733-4744, 2008.

24. Kim SJ, Nakayama S, Miyoshi Y, Taguchi T, Tamaki Y, Matsushima T, Torikoshi Y, Tanaka S, Yoshida T, Ishihara $\mathrm{H}$ and Noguchi S: Determination of the specific activity of CDK1 and CDK2 as a novel prognostic indicator for early breast cancer. Ann Oncol 19: 68-72, 2008.

25. Hansel DE, Dhara S, Huang RC, Ashfaq R, Deasel M, Shimada Y, Bernstein HS, Harmon J, Brock M, Forastiere A, et al: CDC2/CDK1 expression in esophageal adenocarcinoma and precursor lesions serves as a diagnostic and cancer progression marker and potential novel drug target. Am J Surg Pathol 29: 390-399, 2005

26. Chang JT, Wang HM, Chang KW, Chen WH, Wen MC, Hsu YM, Yung BY, Chen IH, Liao CT, Hsieh LL and Cheng AJ: Identification of differentially expressed genes in oral squamous cell carcinoma (OSCC): Overexpression of NPM, CDK1 and NDRG1 and underexpression of CHES1. Int J Cancer 114: 942-949, 2005.

27. Thoms HC, Dunlop MG and Stark LA: p38-mediated inactivation of cyclin D1/cyclin-dependent kinase 4 stimulates nucleolar translocation of RelA and apoptosis in colorectal cancer cells. Cancer Res 67: 1660-1669, 2007.

28. Huang WW, Ko SW, Tsai HY, Chung JG, Chiang JH, Chen KT, Chen YC, Chen HY, Chen YF and Yang JS: Cantharidin induces $\mathrm{G} 2 / \mathrm{M}$ phase arrest and apoptosis in human colorectal cancer colo 205 cells through inhibition of CDK1 activity and caspase-dependent signaling pathways. Int J Oncol 38 1067-1073, 2011 .

29. Soria JC, Jang SJ, Khuri FR, Hassan K, Liu D, Hong WK and Mao L: Overexpression of cyclin B1 in early-stage non-small cell lung cancer and its clinical implication. Cancer Res 60: 4000-4004, 2000.

30. Koon N, Schneider-Stock R, Sarlomo-Rikala M, Lasota J, Smolkin M, Petroni G, Zaika A, Boltze C, Meyer F, Andersson L, et al: Molecular targets for tumour progression in gastrointestinal stromal tumours. Gut 53: 235-240, 2004.

31. Ding $\mathrm{K}, \mathrm{Li} \mathrm{W}, \mathrm{Z}$ ou Z, Zou X and Wang C: CCNB1 is a prognostic biomarker for ER+ breast cancer. Med Hypotheses 83: 359-364, 2014.

32. Yang IP, Tsai HL, Hou MF, Chen KC, Tsai PC, Huang SW, Chou WW, Wang JY and Juo SH: MicroRNA-93 inhibits tumor growth and early relapse of human colorectal cancer by affecting genes involved in the cell cycle. Carcinogenesis 33: 1522-1530, 2012.

33. Scintu M, Vitale R, Prencipe M, Gallo AP, Bonghi L, Valori VM, Maiello E, Rinaldi M, Signori E, Rabitti C, et al: Genomic instability and increased expression of BUB1B and MAD2L1 genes in ductal breast carcinoma. Cancer Lett 254: 298-307, 2007.

34. Shichiri M, Yoshinaga K, Hisatomi H, Sugihara K and Hirata Y: Genetic and epigenetic inactivation of mitotic checkpoint genes hBUB1 and hBUBR1 and their relationship to survival. Cancer Res 62: 13-17, 2002.
35. Yuan B, Xu Y, Woo JH, Wang Y, Bae YK, Yoon DS, Wersto RP, Tully E, Wilsbach K and Gabrielson E: Increased expression of mitotic checkpoint genes in breast cancer cells with chromosomal instability. Clin Cancer Res 12: 405-410, 2006.

36. Andersen CL, Christensen LL, Thorsen K, Schepeler T, Sørensen FB, Verspaget HW, Simon R, Kruhøffer M, Aaltonen LA, Laurberg S and Ørntoft TF: Dysregulation of the transcription factors SOX4, CBFB and SMARCC1 correlates with outcome of colorectal cancer. Br J Cancer 100: 511-523, 2009.

37. Huang YW, Liu JC, Deatherage DE, Luo J, Mutch DG, Goodfellow PJ, Miller DS and Huang TH: Epigenetic repression of microRNA-129-2 leads to overexpression of SOX4 oncogene in endometrial cancer. Cancer Res 69: 9038-9046, 2009.

38. Wang H, Mannava S, Grachtchouk V, Zhuang D, Soengas MS, Gudkov AV, Prochownik EV and Nikiforov MA: c-Myc depletion inhibits proliferation of human tumor cells at various stages of the cell cycle. Oncogene 27: 1905-1915, 2008.

39. Takatsuno Y, Mimori K, Yamamoto K, Sato T, Niida A, Inoue H, Imoto S, Kawano S, Yamaguchi R, Toh H, et al: The rs6983267 SNP is associated with MYC transcription efficiency, which promotes progression and worsens prognosis of colorectal cancer. Ann Surg Oncol 20: 1395-1402, 2013.

40. Sampson VB, Rong NH, Han J, Yang Q, Aris V, Soteropoulos P, Petrelli NJ, Dunn SP and Krueger LJ: MicroRNA let-7a down-regulates MYC and reverts MYC-induced growth in Burkitt lymphoma cells. Cancer Res 67: 9762-9770, 2007.

41. Gao P, Tchernyshyov I, Chang TC, Lee YS, Kita K, Ochi T, Zeller KI, De Marzo AM, Van Eyk JE, Mendell JT and Dang CV: c-Myc suppression of $\mathrm{miR}-23 \mathrm{a} / \mathrm{b}$ enhances mitochondrial glutaminase expression and glutamine metabolism. Nature 458: $762-765,2009$.

42. Chen Z, Zeng H, Guo Y, Liu P, Pan H, Deng A and Hu J: miRNA-145 inhibits non-small cell lung cancer cell proliferation by targeting c-Myc. J Exp Clin Cancer Res 29: 151, 2010.

43. Smith MJ, Culhane AC, Donovan M, Coffey JC, Barry BD, Kelly MA, Higgins DG, Wang JH, Kirwan WO, Cotter TG and Redmond HP: Analysis of differential gene expression in colorectal cancer and stroma using fluorescence-activated cell sorting purification. Br J Cancer 100: 1452-1464, 2009.

44. Vazquez A, Bond EE, Levine AJ and Bond GL: The genetics of the p53 pathway, apoptosis and cancer therapy. Nat Rev Drug Discov 7: 979-987, 2008.

45. Al-Kuraya K, Novotny H, Bavi P, Siraj AK, Uddin S, Ezzat A, Sanea NA, Al-Dayel F, Al-Mana H, Sheikh SS, et al: HER2, TOP2A, CCND1, EGFR and C-MYC oncogene amplification in colorectal cancer. J Clin Pathol 60: 768-772, 2007.

46. Huang HY, Illei PB, Zhao Z, Mazumdar M, Huvos AG, Healey JH, Wexler LH, Gorlick R, Meyers P and Ladanyi M: Ewing sarcomas with p53 mutation or p16/p14ARF homozygous deletion: A highly lethal subset associated with poor chemoresponse. J Clin Oncol 23: 548-558, 2005.

47. Choi EJ and Kim GH: Apigenin causes G(2)/M arrest associated with the modulation of p21(Cip1) and Cdc2 and activates p53-dependent apoptosis pathway in human breast cancer SK-BR-3 cells. J Nutr Biochem 20: 285-290, 2009.

48. Li CJ, Li RW, Wang YH and Elsasser TH: Pathway analysis identifies perturbation of genetic networks induced by butyrate in a bovine kidney epithelial cell line. Funct Integr Genomics 7: 193-205, 2007.

49. Chen J, Feilotter HE, Paré GC, Zhang X, Pemberton JG, Garady C, Lai D, Yang X and Tron VA: MicroRNA-193b represses cell proliferation and regulates cyclin D1 in melanoma. Am J Pathol 176: 2520-2529, 2010.

50. Xia W, Li J, Chen L, Huang B, Li S, Yang G, Ding H, Wang F, Liu N, Zhao Q, et al: MicroRNA-200b regulates cyclin D1 expression and promotes S-phase entry by targeting RND3 in HeLa cells. Mol Cell Biochem 344: 261-266, 2010.

51. Liu X, Lv XB, Wang XP, Sang Y, Xu S, Hu K, Wu M, Liang Y, Liu P, Tang J, et al: MiR-138 suppressed nasopharyngeal carcinoma growth and tumorigenesis by targeting the CCND1 oncogene. Cell Cycle 11: 2495-2506, 2012.

52. Schultz J, Lorenz P, Gross G, Ibrahim S and Kunz M: MicroRNA let-7b targets important cell cycle molecules in malignant melanoma cells and interferes with anchorage-independent growth. Cell Res 18: 549-557, 2008. 